\title{
Corrigendum: How dark matter came to matter
}

J. G. de Swart, G. Bertone and J. van Dongen

Nature Astronomy 1, 0059 (2017); published 2 March 2017; corrected 6 March 2017.

In the version of this Review Article originally published George Ellis was mistakenly named as John Ellis. This has been corrected in all versions of the Review Article. 